\title{
PANDEMIA, POLÍTICAS PÚBLICAS Y SECTORES VULNERABLES: UN ANÁLISIS DEL INGRESO FAMILIAR DE EMERGENCIA EN ARGENTINA
}

\section{Lucía Kaplan ${ }^{1}$}

luciakaplan@gmail.com

Universidad Nacional de Rosario y CONICET, Argentina.

\section{Andrea Delfino ${ }^{2}$}

andelfino@yahoo.com.ar

Universidad Nacional del Litoral y Universidad Nacional de Rosario, Argentina.

La expansión vertiginosa del coronavirus a partir de marzo de 2020, conllevó en Argentina el confinamiento de la población como medida sanitaria para contener la pandemia. Las medidas de aislamiento social impusieron restricciones a la circulación y a la asistencia a los lugares de trabajo. Este nuevo escenario de pandemia se desplegó sobre un mercado de trabajo que acumulaba fragilidades y tensiones de largo plazo y esas tensiones se habían profundizado en los últimos años. Como consecuencia de esta situación, este artículo se centra en describir y analizar el programa Ingreso Familiar de Emergencia, las características de sus beneficiarios y sus impactos desde la perspectiva de la intervención social del Estado haciendo uso de información pública y documentos oficiales. Dicha política de transferencia de renta fue direccionada a los sectores más vulnerables -fundamentalmente trabajadores informales- y constituye el programa más importante del período en cuanto al alcance poblacional.

Palabras Claves: pandemia por coronavirus - sectores vulnerables - transferencias de renta - Argentina

\footnotetext{
Licenciada en Ciencia Política, Universidad Nacional de Rosario, Argentina. Becaria Doctoral Conicet.

2 Doctora en Ciencia Política, Universidad Nacional de Rosario,Argentina. Profesora de la Universidad Nacional del Litoral y de la Universidad Nacional de Rosario. Investigadora del IHUCSO-Litoral, Argentina.
} 
PANDEMIC, PUBLIC POLICIES AND VULNERABLE SECTORS: AN ANALYSIS OF THE EMERGENCY FAMILY INCOME IN ARGENTINA

The dramatic spread of coronavirus since March 2020 has led to the confinement of Argentine population as a health measure in order to contain the pandemic. Social isolation measures have restricted people's movement and attendance at their workplaces. This new pandemic scenario was developed when the labor market had been accumulating long-term fragilities and tensions, and these tensions had been increasing in the last few years. As a consequence of this situation, this article is centered in the description and analysis of the Emergency Family Income program, the characteristics of its beneficiaries and its impacts from the perspective of social state intervention, using public information and official documents. This income transfer program was directed towards the most vulnerable sectors - mainly informal workers- and constitutes the most important program of the period as regards the amount of population reached.

Key words: coronavirus pandemic - vulnerable sectors - income transfers - Argentina 


\section{Introducción}

La expansión mundial de coronavirus (COVID-19) a partir de diciembre de 2019 ha instalado una nueva realidad socioeconómica de consecuencias aún imprevisibles. La rápida propagación del virus, hasta el momento desconocido, y la severidad con que se manifestó en innumerables casos, obligó a los diferentes gobiernos a tomar medidas inéditas de prevención que, en la mayoría de los países, consistieron en políticas de aislamiento social.

Estas medidas han permitido en muchos países disminuir los contagios, evitar el colapso de los sistemas sanitarios y preservar vidas; sin embargo, al mismo tiempo, el confinamiento de gran parte de la población mundial ha paralizado la actividad económica ${ }^{3}$ generando una contracción que se estima la más severa desde la Gran Depresión (CEPAL, 2020).

En este contexto, la Argentina no ha sido una excepción. Las medidas de aislamiento obligatorio que se implementaron en el marco de la emergencia sanitaria pusieron de manifiesto la vulnerabilidad del tejido social en este país, como así también la capacidad estatal para generar políticas (Campana y Ferrari Mango, 2021) que permitan contener los efectos sociales y económicos de la pandemia.

En este sentido, el objetivo de este artículo es describir y analizar las principales características del diseño del Ingreso Familiar de Emergencia (IFE) ${ }^{4}$ y de sus beneficiarios y beneficiarias desde la perspectiva de la intervención social del Estado. Esta medida excepcional implementada por el Gobierno Nacional tuvo por objetivo ofrecer una prestación económica a aquellas familias argentinas que sufrieron la pérdida o disminución de sus ingresos como consecuencia de la situación de emergencia sanitaria generada por el COVID-19.

El análisis de esta política en particular se debe a dos motivos: en primer lugar, el IFE fue una iniciativa de gran trascendencia que alcanzó a casi 9 millones de trabajadores de la economía informal, monotributistas sociales, monotributistas de las categorías inferiores, trabajadoras y trabajadores de casas particulares y personas que se encontraban desempleadas. Por su envergadura y por el volumen de población alcanzado puede considerársela la política insignia de este período. En segundo lugar, el IFE permitió obtener información sobre un conjunto heterogéneo de población que nunca antes había sido comprendido por políticas de este tipo; de esta forma, "así como la Asignación Universal por Hijo (AUH) a su tiempo, se ofrece como una nueva plataforma institucional para la reflexión sobre el carácter y el alcance de las transferencias de ingresos en la postpandemia” (Arcidíacono y Gamallo, 2020:13).

3 CEPAL está refiriendo exclusivamente a la actividad económica producida mercantilmente, ya que el tiempo dedicado al trabajo doméstico y de cuidados no remunerado se ha expandido.

4 Decreto de Necesidad y Urgencia 310/2020. 
Teniendo en cuenta las características de esta política, una de las hipótesis que estructurará estas páginas es que el alcance y la magnitud del IFE puede explicarse, por un lado, por la existencia de desigualdades estructurales en materia de condiciones de trabajo e ingresos (Salvia y Vera, 2015) y, por otra parte, por el agravamiento y deterioro de estas condiciones durante el gobierno de la Alianza Cambiemos ${ }^{5}$ (2015-2019), (Pastrana y Trajtemberg, 2020; Manzanelli, Calvo y Basualdo, 2020; CEDYT, 2020b; Ernst y Mourelo, 2020).

En esta dirección, el artículo se centra en un primer lugar en describir el proceso de profundización de las desigualdades y aumento de los niveles de precarización en el empleo que se evidenciaron en el mercado de trabajo en el período 201619. En segundo lugar, describe el repertorio de políticas públicas desplegadas por el gobierno argentino en el marco de la emergencia sanitaria desde la perspectiva de la intervención social del Estado. Finalmente, reconstruye a través del uso de información pública y documentos oficiales las características y los impactos del IFE, el programa de mayor envergadura en cuanto a la magnitud de perceptores y/o beneficiarios del período. En tanto, las consideraciones finales dan cuenta de los desafíos que implica la consolidación de un colectivo cada vez más amplio de trabajadores/as vulnerables que no logran acceder de forma sostenida a las protecciones que garantiza el sistema de seguridad argentino.

\section{El mercado de trabajo argentino: una mirada desde las vulnerabilidades persistentes}

Los impactos de la emergencia sanitaria sobre los principales indicadores socioeconómicos son innegables. Los datos correspondientes al último trimestre de 2020 que se desprenden de la Encuesta Permanente de Hogares (EPH) -realizada trimestralmente por el Instituto Nacional de Estadística y Censos (INDEC)- evidencian un empeoramiento de todos los indicadores. Sin embargo, se puede observar una correspondencia entre la situación de extrema vulnerabilidad que exhiben al día de hoy ciertos indicadores sociales y las características que fue adquiriendo el mercado de trabajo en nuestro país antes de la irrupción del COVID-19

Si bien es preciso mencionar que las desigualdades laborales constituyen un rasgo estructural del mercado de trabajo en Argentina, es posible distinguir que, durante los cuatro años previos a la emergencia sanitaria, los indicadores laborales mostraron un desmejoramiento que puede explicarse, entre otras cosas, por las orientaciones

5 La Alianza Cambiemos está constituida por el partido Propuesta Republicana (PRO) como socio principal, junto a sectores de la Unión Cívica Radical (UCR) y la Coalición Cívica (CC). Luego de 12 años de gobiernos kirchneristas (Néstor Kirchner 2003-2007; Cristina Fernández de Kirchner 2007-2015) llega al poder Mauricio Macri, referente de esta alianza política, que gobernó la Argentina durante el período 2015 - 2019. 
políticas y económicas que la Alianza Cambiemos imprimió como política de Estado, tras haber ganado, en diciembre de 2015, la presidencia de la Nación argentina.

El triunfo electoral de la nueva coalición dominante supuso la instauración de un nuevo bloque de poder al interior del Estado que reunió a gran parte de los sectores políticos y económicos que fueron desplazados durante las tres gestiones consecutivas del Frente de Todos, liderado primero por Néstor Kirchner y luego por Cristina Fernández.

En este sentido, a partir del 2016 comienzan a tomar preponderancia aquellos actores vinculados con el capital financiero, agrario y de servicios que buscaron imponer un proyecto político y económico que cristalice la nueva correlación de fuerza (Barrera Insua, Pérez, 2020; Delfino y Kaplan, 2019). El resultado de esta nueva configuración del poder fue el desarrollo de la valorización financiera como eje central del nuevo patrón de acumulación del capital interno (CIFRA-FLACSO, 2016) y la consecuente redefinición del rol del Estado para garantizar el desarrollo de las empresas como comunidades productivas, innovadoras, eficientes y competitivas.

Para poder dar cuenta del impacto de este nuevo modelo de acumulación, presentaremos a continuación un breve análisis de la evolución de la tasa de actividad, de empleo y desempleo de 2015 a 2020, como así también realizaremos una caracterización de la calidad del empleo con el fin de proporcionar una serie de datos que nos permitan comprender la particular situación de vulnerabilidad que atraviesa la Argentina por la crisis del COVID-19.

\section{Gráfico $N^{\circ} 1$}

Tasa de actividad, empleo y desempleo 2015-2020

Total de aglomerados urbanos (en \% sobre población económicamente activa)

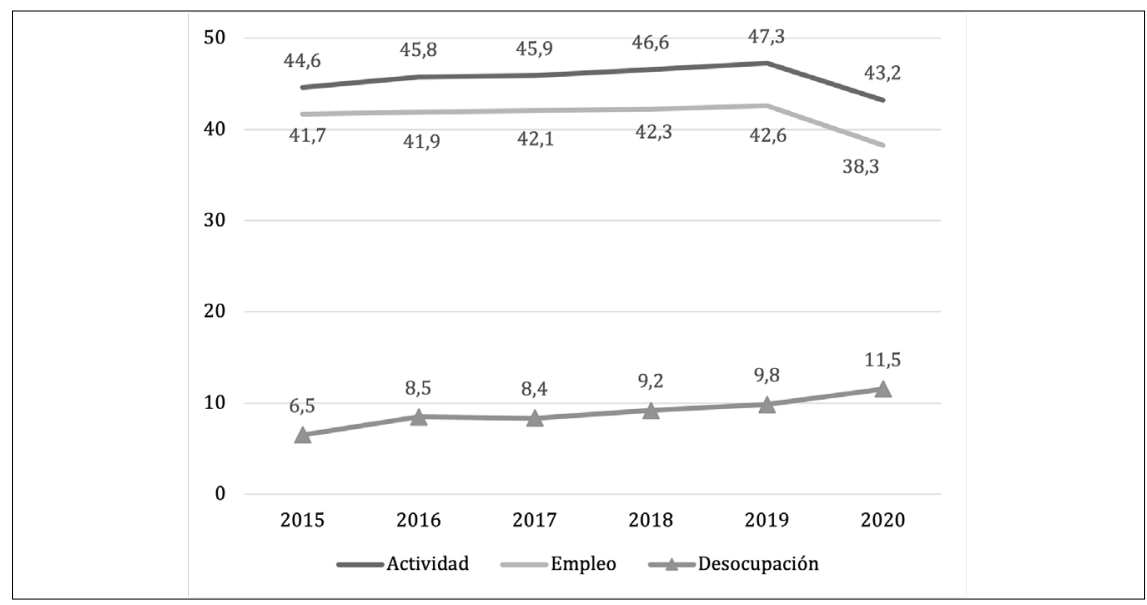

Fuente: Elaboración propia sobre la base de EPH-INDEC 
En primer lugar, es preciso aclarar que para construir un escenario más detallado se incorporan los promedios anuales correspondientes al 2015, debido a que estas tasas expresan la situación del mercado de trabajo durante el último año de la gestión de Cristina Fernández de Kirchner y resultan un punto de partida para comprender la evolución posterior y el impacto de la pandemia sobre los indicadores que se analizan.

Se puede observar, en primer lugar, que la tasa de actividad durante los 4 años de la gestión de Cambiemos ha sostenido niveles de crecimiento constantes con respecto al 2015, mostrando una mayor evolución a partir del 2018.

Este dato cobra relevancia si se tiene en cuenta que, a principios de ese año, se produce una fuerte devaluación de la moneda que, entre otros aspectos, provocó una intervención del Banco Central en el manejo de la tasa de interés para restringir la cantidad de dinero en circulación y disminuir la demanda de dólares (Barrera Insua y Pérez, 2020). El efecto directo de esta situación fue una contracción en la actividad económica, como así también, un aumento de precios que impactó sobre un salario real cada vez más deteriorado.

El deterioro salarial fue particularmente intenso desde el segundo cuatrimestre de 2018 es decir, luego de que la corrida cambiaria impactara sobre el nivel de precios y que la situación de deuda condujera a tomar un préstamo del FMI.Así, en 2019 el salario real era $12,5 \%$ inferior al promedio de 2015 y en diciembre de 2019 la caída llegó al 14, 8\% (CIFRA, 2020)

En este contexto, se considera que el aumento en la tasa de actividad a partir de 2018 se debe, fundamentalmente, a una mayor participación laboral de las mujeres que ingresan al mercado de trabajo para subsidiar la caída de ingresos del hogar en este contexto recesivo (Díaz Langou et al., 2019).

El efecto de la pandemia sobre este indicador permite observar que una de las principales expresiones de la crisis laboral tras la emergencia sanitaria fue la caída en 4 puntos porcentuales de la tasa de actividad durante el 2020; esto significa que, al finalizar ese año, alrededor de 3 millones y medio de personas dejaron de tener trabajo o de buscarlo activamente.

En cuanto a la tasa de empleo, se pueden observar a lo largo del período 2015/ 20 dos momentos diferenciados: en primer lugar, desde el 2016 al 2019 este indicador se mantiene de forma constante, sin embargo, su crecimiento fue mucho más moderado (1,5\%) que el de la tasa de actividad (3\%); esta situación indica que durante la gestión de Mauricio Macri la dinámica de creación de nuevos puestos de trabajo estuvo por detrás del crecimiento de la población activa, lo que justifica el sostenido aumento de la tasa de desempleo.

Con respecto al descenso de los niveles de empleo a partir del 2020, se puede inferir que, a pesar de todas las medidas llevadas a cabo por el gobierno nacional 
para sostener los puestos de trabajo, esta caída, de aproximadamente el 4\%, expresa la pérdida de aquellos empleos vinculados de forma más cercana con algún tipo de vulnerabilidad, nos referimos particularmente a la situación de los trabajadores independientes como así también a los informales ${ }^{6}$.

Con respecto a la tasa de desempleo, es preciso distinguir algunos aspectos; en primer lugar, es importante tener en cuenta que durante el año 2015 los desocupados habían descendido al 6,5\% de la Población Económicamente Activa (PEA), lo que constituye, según CIFRA (2017), el valor más bajo de las últimas tres décadas. Sin embargo, estos porcentajes no lograron sostenerse durante el 2016, cuando se produjo un aumento del $2 \%$ de la desocupación. Esta situación puede explicarse por la contracción de la actividad económica en un 2,2\%, como consecuencias de una política de apertura comercial que impactó negativamente sobre el entramado productivo local, como así también por la contracción en el sector de la construcción, empujada por la caída en el gasto en obra pública.A su vez, "los incentivos para la especulación financiera generados a partir de la política de emisión de letras del BCRA (LEBAC) implicaron un desaliento adicional para las inversiones productivas en general"' (CIFRA, 2017; 4). En consecuencia, la tasa de desocupación creció de forma constante hasta llegar a los dos dígitos en el segundo trimestre del 2019 (10,6 por ciento); esto significa que, para finales de 2019, cerca de 1,2 millones de personas buscaban empleo en Argentina.

Un párrafo que merece especial atención es la vulnerabilidad laboral que experimentan determinados sectores. En este sentido, si analizamos la tasa de desempleo por género y edad se identifica que, de forma constante a lo largo de todo el período, para las mujeres y los jóvenes la situación resulta más alarmante. Si tomamos los promedios correspondientes al 2019, podemos observar que la tasa de desocupación para las mujeres es de 10,6\%, mientras que para los varones estos valores se sitúan en el 9,1\%. Lo mismo sucede con la población joven, para las mujeres de 14 a 29 años, la desocupación alcanza al 18,6\%, dos puntos porcentuales más que para los varones del mismo rango etario y un $13 \%$ más que para los desocupados mayores de 29 años.

Por último, es preciso mencionar que para el 2020, la tasa de desempleo aumentó casi 2 puntos porcentuales, sin embargo, como pudimos constatar a partir de los datos de la EPH, la desocupación constituye una realidad que viene creciendo de forma sostenida desde el 2016 y como consecuencia de la pandemia, no hizo más que profundizarse.

Además de los altos niveles de desempleo, se pueden identificar algunos otros aspectos vinculados a la calidad del trabajo que posicionan a Argentina en una situación de especial vulnerabilidad ante el impacto de la crisis provocada por la COVID-19 (Ernst y Mourelo, 2020).

Siguiendo a Tokman (1997) consideramos que la informalidad laboral no es una problemática que se circunscribe solamente las unidades productivas informales, sino que el empleo no registrado forma parte de las estrategias empresariales por bajar costos laborales. 
Con respecto a la calidad del trabajo, merece especial atención la evolución en el tipo de empleo que se creó en el país durante el período 2016/2019. En este sentido, es posible afirmar que en Argentina siguen predominando los asalariados registrados como categoría ocupacional que reúne a la mayor parte de la población empleada. Según los datos de SIPA (Sistema Integrado Previsional Argentino) presentados por el Ministerio de Trabajo y Seguridad Social de Argentina, para el último trimestre de 2019, existían más de 12 millones de personas inscriptas bajo este régimen.

Si analizamos la dinámica del empleo dentro del sector de asalariados registrados, podemos observar que durante los años 2016-2019, se produjo un retroceso en la creación de puestos de trabajo dentro del ámbito privado. En el año 2016, se perdió el 0,6\% de los empleos asalariados privados con respecto al año 2015, y luego de ese año, se observa una pequeña recuperación en el 2017, que no logró sostenerse en los años posteriores (Gráfico No 2). Es preciso mencionar que, a finales del 2015, la categoría asalariado privado representaba al 53\% del total de asalariados registrados, mientras que, al finalizar el 2019, este sector de trabajadores ocupaba el 50\% del total.

En contraposición a esta situación, se destaca el desempeño del empleo público que evidenció, sobre todo a partir del año 2016, una sostenida creación de puestos de trabajo. Es preciso mencionar que dentro de este ámbito ocurrieron dos procesos paralelos. Por un lado, se llevaron a cabo numerosos despidos que obedecieron a cuestiones políticas y al desmantelamiento de numerosos programas estatales vinculados con la gestión de gobierno anterior y, por otra parte, se incorporó una importante cantidad de empleados públicos, en todos los niveles, con el objetivo de incluir personal afin al nuevo gobierno dentro de la Administración Pública (Astarita y De Piero, 2017; Barrera Insua y Pérez, 2020). 


\section{Gráfico $\mathrm{N}^{\circ} 2$}

Variación en los trabajos registrados según modalidad, 2016 - 2019

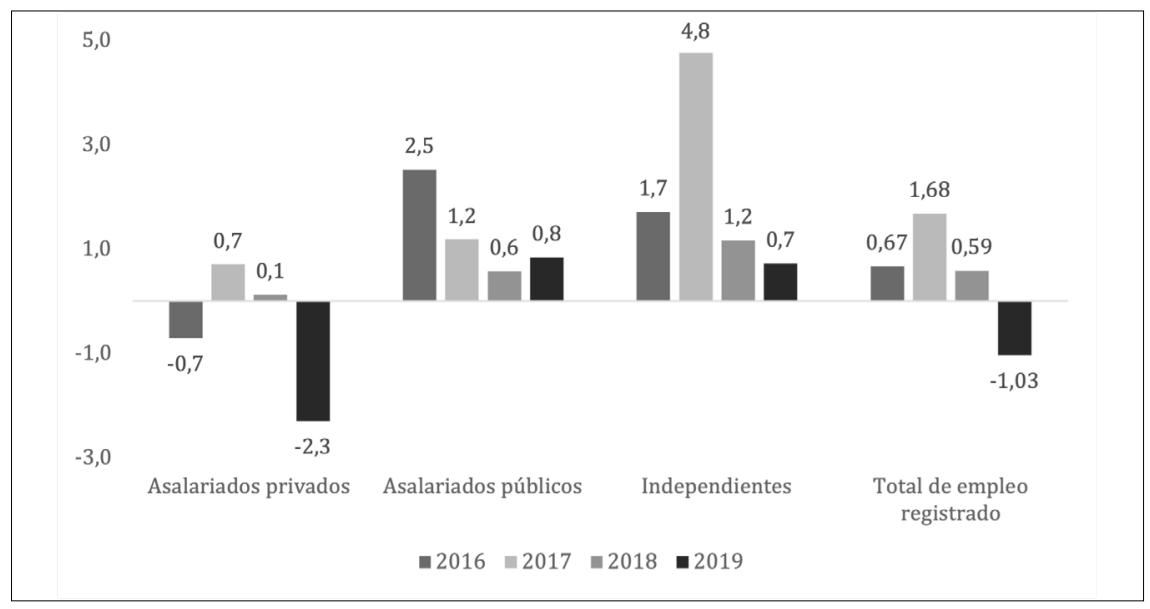

Fuente: elaboración propia sobre la base de información de SIPA publicada por el Ministerio de Trabajo, Empleo y Seguridad Social

Así mismo, el Gráfico $\mathrm{N}^{\circ} 2$ permite observar que, dentro de las ocupaciones registradas, la modalidad que más se incrementó entre 2016 y 2019 fue la de trabajadores independientes, que engloba a quienes están registrados como autónomos, monotributistas y monotributistas sociales ${ }^{7}$. Se trata de un conjunto muy heterogéneo de trabajadores en términos de estabilidad y de ingresos, que se caracteriza por tener ingresos promedio más bajos e inseguros respecto de los que corresponden a los trabajadores asalariados (CIFRA, 2017).

Lo relevante de esta categoría ocupacional, es que aglutina a trabajadores que, mediante alguno de los regímenes tributarios vigentes, realiza aportes a la seguridad social y que, en la mayoría de los casos, se encuentran bajo una relación de dependencia encubierta. Estos trabajadores, según un informe de UMET-CITRA (2019) se podrían definir como asalariados para los cuales se encubre la relación de dependencia con su empleador a partir de su registro en el régimen monotributista. Así, este tipo de relación de trabajo sustituye al empleo formal, al mismo tiempo que

7 En Argentina el Monotributo es un Sistema de Régimen Simplificado que concentra en un único tributo el componente previsional (aportes de jubilación y obra social) y el impositivo (donde se separa en categorías basándose en los ingresos brutos obtenidos según facturación, la superficie afectada a la actividad y la energía eléctrica consumida). De este modo, en un único pago mensual, los monotributistas pueden cumplir con los pagos obligados de impuesto a las ganancias, IVA, aportes jubilatorios y al Sistema Nacional del Seguro de Salud. En tanto el Monotributo Social es una categoría tributaria permanente que reconoce la realización de actividades productivas, comerciales y de servicios por parte de la población en situación de vulnerabilidad social. 
aumenta la precarización, en tanto estos trabajadores cuentan con menos derechos laborales.

En síntesis, puede destacarse que durante el período 2016-2019, aumentó la cantidad de trabajadores independientes alcanzando a finales de 2019 a más de 2.397.000 personas y disminuyó la de trabajadores en relación de dependencia (9.208,9 millones de trabajadores de este sector). Dentro de este último grupo, se achicó a su vez el peso de los empleados del sector privado, como consecuencia de la escasa generación de puestos de trabajo en esta área.

Como puede observarse a partir de los datos precedentes, durante el período 2016-2019, se consolidaron y profundizaron ciertas tendencias del mercado laboral argentino que tienen que ver con los altos niveles de desempleo y la existencia, cada vez más pronunciada, de trabajadores vinculados a modalidades de empleo precarias.

\section{Pandemia y políticas públicas}

Existe un acuerdo generalizado entre las diferentes perspectivas sobre las políticas públicas que conduce a afirmar que las mismas expresan la relación entre la sociedad y el Estado e indican un balance específico de relaciones de poder que está en íntima relación con el régimen político y con un proyecto de dirección política y dirección ideológica del Estado (Medellín, 2004, García Linera, 2010; Vilas, 2010, Oszlak, 1980). En este sentido, las políticas públicas pueden ser comprendidas como "el conjunto de objetivos, decisiones y acciones que lleva a cabo un gobierno para solucionar los problemas que en un momento determinado los ciudadanos y el propio gobierno consideran prioritarios” (Tamayo Sáenz, 1997:281).

Dentro de este marco general la noción de "intervención social del Estado" (Minujín y Cosentino, 1993) posibilita abordar específicamente aquel conjunto de políticas que afectan las condiciones de vida y de trabajo de la población, poniendo en relación las políticas que operan tanto en la distribución primaria del ingreso como en la distribución secundaria y a la vez situarlas en el contexto económico, social, político y cultural en el que se despliegan.

La administración de Alberto Fernández que asumió en diciembre 2019 implementó, en los primeros meses de gestión, una serie de medidas orientadas a contener la crítica situación socioeconómica heredada. Estas políticas tuvieron como objetivo recomponer los ingresos de los/as jubilados/as y beneficiarios/as de la Asignación Universal por Hijo (AUH) y Asignación Familiar por Embarazo para la Protección Social $(\mathrm{AE})^{8}$ a través de un bono e incrementos en los montos de los haberes, como

8 La AUH es una asignación destinada a niños/as menores de 18 años y personas con discapacidad sin límite de edad. Su principal objetivo es garantizar un ingreso universal a los niños en situación de vulnerabilidad socio-económica, a la vez que asegurar la asistencia a la escuela, la realización de controles periódicos de salud y el cumplimiento del calendario de vacunación obligatorio. La 
así también se acordaron aumentos salariales para trabajadores del sector privado y público a cuenta de futuras paritarias y se estableció la obligación de pagar doble indemnización en caso de despido por 180 días.

Durante estos primeros meses también se creó el Programa Alimentar, cuyo objetivo es asegurar la alimentación de los/as niños/as de hasta seis años beneficiarios/as de la AUH o AE, y se congelaron las tarifas de los servicios públicos.

En ese contexto, la irrupción de la pandemia y el aislamiento social obligatorio llevaron al Estado a profundizar las medidas que venía implementando, como así también a adoptar un conjunto de políticas con el fin de preservar los empleos, los ingresos y evitar el cierre de empresas.

Estas medidas se pueden clasificar en tres grandes grupos (Manzanelli, Calvo y Basualdo, 2020). En primer lugar, se pueden identificar aquellas políticas destinadas a la asignación de ingresos focalizadas principalmente en los sectores más vulnerables. Dentro de este grupo se destaca:

- La emisión de un bono extraordinario para jubilados y pensionados que cobran la mínima y para los beneficiarios de AUH y AE.

- El Ingreso Familiar de Emergencia (IFE) destinado a trabajadores/as informales, desocupados/as, personal doméstico, monotributistas sociales y categoría A y B.

- Refuerzo en el Programa Argentina contra el Hambre (Tarjeta Alimentar; Comedores escolares, comunitarios y merenderos).

Con respecto al segundo grupo de políticas, podemos distinguir aquellas iniciativas orientadas a contener precios de productos, alquileres y tarifas de servicios públicos. Dentro de este grupo se distinguen las siguientes regulaciones:

- Suspensión de corte por falta de pago de servicios públicos incluso telefonía e internet a titulares de la AUH y AE, jubilados/as, pensionados/as, perceptores/ as seguro de desempleo y a usuarios/as electrodependientes, monotributistas sociales, empleadas domésticas, etc. Esta medida estuvo vigente hasta el 31 de diciembre de 2020.

- Prohibición de desalojos de viviendas alquiladas por falta de pago y prórroga de contratos de alquiler vencidos, vigente hasta el 31 de marzo de 2021.

- Congelamiento de cuotas de créditos UVA según cuota valor marzo hasta el 31 de enero de 2021.

AE es una transferencia monetaria que se abona mensualmente y está destinada a aquellas mujeres embarazadas a partir de la decimosegunda semana de gestación, hasta el nacimiento o interrupción del estado de embarazo. Pueden acceder a esta asignación trabajadoras informales, servicio doméstico y desempleadas. 
- Congelamiento de tarifas de tv paga, internet y telefonía móvil y fija hasta el 31 de diciembre de 2020 .

- Período de gracia para el pago de cuotas de créditos ANSES.

Por último, siguiendo el criterio de clasificación ya mencionado, se encuentran todas aquellas políticas destinadas, principalmente, a la producción y en menor medida a la salud, ciencia y obra pública. Dentro de este paquete de medidas, se distingue por la magnitud de su alcance, el programa de Asistencia al Trabajo y la Producción (ATP). Esta política, que estuvo vigente hasta finales de 2020, tuvo diferentes aristas que abarcaron tanto la postergación o reducción de contribuciones patronales a empleadores de los sectores más afectados (actividades de esparcimiento, turismo, transporte de pasajeros y hoteles, etc.), como así también el pago de una asignación complementaria al salario a trabajadores de dichos sectores. Según los datos proporcionados por el Ministerio de Desarrollo Productivo, esta iniciativa implicó el desembolso de ARS 370.240 millones que aseguraron cierto nivel de ingresos para casi la mitad de la población trabajadora registrada en relación de dependencia; es decir, se trató de una medida que alcanzó a más de 3 millones de trabajadores.

Dentro de este conjunto, se pueden reconocer otras iniciativas tales como:

- Préstamos a tasa del 0\% para personas adheridas al Régimen Simplificado para Pequeños Contribuyentes (monotributo categorías C, D, E) y para trabajadores autónomos.

- Prohibición de despidos y suspensiones con excepción de las previstas en el Art. 223 bis de la Ley de Contrato de Trabajo.

- Créditos a tasa de 24\% fija anual para Pymes con tres meses de gracia.

- Bonos especiales para trabajadores de la salud, de seguridad y de defensa.

- Creación del Fondo de Garantía para la Micro, Pequeña y Mediana Empresa

- Licencias y trabajo remoto para grupos de riesgo, embarazadas y mayores de 60.

- $\quad$ Reconocimiento de la COVID-19 como accidente de trabajo por ART.

En términos generales, se podría afirmar que las políticas implementadas a partir de la pandemia permitieron garantizar ingresos básicos a casi 11 millones de personas. Si a ese colectivo se le suman los 3,2 millones de trabajadores que se desempeñan en el sector público, los 6,9 millones de jubilados y pensionados y los 120 mil que perciben el seguro de desempleo, se podría afirmar que el Estado a través de estas iniciativas logró contener y sostener los ingresos de casi 21 millones de personas (CETyD, 2020a). 


\section{El Ingreso Familiar de Emergencia: su diseño y características}

El Ingreso Familiar de Emergencia ${ }^{9}$ (IFE, en adelante) fue creado en Argentina de forma simultánea con el establecimiento de medidas de confinamiento como estrategia sanitaria para contener la pandemia de COVID-19. Así, el Aislamiento Social, Preventivo y Obligatorio (ASPO) supuso la imposición de restricciones a la circulación y a la asistencia a los lugares de trabajo, excepto para aquellos trabajadores de las consideradas actividades esenciales.

El impacto de esta situación fue prácticamente generalizado pero extremadamente desigual producto de la estructura heterogénea, desigual y fragmentada del mercado de trabajo ya descripta. Tal como señalan Benza y Kessler (2021) para toda América Latina, las mayores pérdidas de empleo se registraron en las actividades de contacto más intensivo y el impacto negativo de la crisis ha sido especialmente acentuado en las actividades informales. A diferencia de otras crisis, con el shock de la pandemia por COVID-19 el sector informal no actuó como refugio de quienes perdieron sus empleos y los trabajadores informales estuvieron entre los más afectados. En este grupo de trabajadores existen muy pocas posibilidades de adoptar el teletrabajo, debido al predominio de actividades que requieren contacto cercano, al tiempo que los trabajadores informales tienen una mayor presencia en los estratos de menores ingresos.

El IFE fue diseñado como una prestación monetaria no contributiva de carácter excepcional, destinado a personas desocupadas, trabajadores informales, monotributistas de las categorías inferiores y trabajadoras de casas particulares (registradas o no) cuyo grupo familiar no tuviera otros ingresos, de entre 18 y 65 años. El programa se abonó a un solo integrante del grupo familiar ${ }^{10}$ y fue compatible con la AUH y AE. Una característica de esta medida es que priorizaba la titularidad de mujeres.

En términos de diseño el programa estaba pensado para focalizar en la población de ingresos inestables, los cuales constituyen el 26\% de la población económicamente activa de Argentina. Además, la mayor parte de los cuentapropistas, desocupados/as, y trabajadores/as informales pertenecen a sectores de bajos ingresos: el 58,8\% son pobres. Así, el programa estaba direccionado a aquellos sectores de la población con mayor grado de vulnerabilidad en términos socio-económicos.

La prestación equivalió a un monto de ARS 10.000, lo que representaba el 59,3\% del Salario Mínimo Vital y Móvil y es sustancialmente menor a la canasta básica

\footnotetext{
Decreto de Necesidad y Urgencia (DNU) N³10/2020
}

10 Por grupo familiar el programa consideraba al compuesto por el o la solicitante, su cónyuge o conviviente y sus hijos menores de 18 años, o sin límite de edad en el caso de hijos con discapacidad. Estaba prevista la consideración de los grupos familiares unipersonales. El componente familiarista es un criterio muy arraigado en las transferencias no contributivas, en oposición al tratamiento individual de las prestaciones contributivas (Arcidíacono y Gamallo, 2020). 
total (delimitación de la denominada línea de pobreza) para un hogar de cuatro miembros que en abril de 2020 rondaba los ARS 42.600. En términos generales podría señalarse que fue un programa destinado a compensar de forma parcial la pérdida o grave disminución de ingresos de las personas afectadas por la situación de emergencia sanitaria.

Según datos de prensa $^{11}$ e información oficial disponible, la estimación inicial del gobierno fue que al programa se destinarían ARS 40 millones y alcanzaría a aproximadamente 3,7 millones de beneficiarios, los cuales corresponderían a 2,2 millones de perceptores de la AHU y 1,5 millones de trabajadores informales. Sin embargo, la cantidad de inscriptos superó ampliamente esas previsiones pasando los 13 millones.

En efecto, la cantidad de solicitantes del programa IFE fue levemente superior a los 13,4 millones de personas, de las cuales 7,1 millones (52,9\%) corresponden a mujeres y 6,3 millones a varones $(47,1 \%)$. Entre los rangos etarios predominan 25 a 34 años con 4,0 millones (30,1\%) y 18 a 24 años con 3,3 millones (25,0\%). Existieron 3.560 solicitantes (menos del 0,1\%) por debajo y poco más de 100 mil $(0,8 \%)$ por encima de la edad requerida (ANSES, 2020).

Los solicitantes realizaron su inscripción de forma virtual y en un momento donde las oficinas de la Administración Nacional de la Seguridad Social, ANSES se encontraban cerradas. Sin embargo, se desplegó un dispositivo de apoyo territorializado basado en modalidades itinerantes móviles, espacios de atención en organizaciones comunitarias y contando con la colaboración de otras órbitas del Estado para dar respuesta -sobre todo- a la población con escasa o nula posibilidad de conectividad.

En relación a la distribución de los solicitantes por situación laboral puede señalarse que el grupo mayoritario era el de los de trabajadores/as informales o desempleados/ as sin Seguro por Desempleo. Un total de 5,5 millones (40,7\%) trabajadores/as informales o desempleados/as se inscribieron para obtener el beneficio, dentro de ese grupo 3,4 millones fueron varones y 2,1 millones mujeres. El segundo grupo representativamente más numeroso en solicitar el IFE fueron beneficiarios de la AUH (2,4 millones de personas, que representa el 18,0\% de los solicitantes). En este grupo - por las propias características de la AUH- predominan las mujeres (2,3 millones vs. 0,1 millones de varones). En tercer lugar, con 1,4 millones (10,2\%) se encuentran las personas solicitantes adheridas al monotributo, donde la distribución es bastante homogénea entre ambos géneros. Finalmente, se encuentran las personas solicitantes que trabajan bajo el Régimen de Casas Particulares (más de 300 mil -2,4\%-) y

11 Wainfeld en Página 12, 20 de abril de 2020. 
quienes perciben el programa PROGRESAR ${ }^{12}$ (128 mil -1,0\%-). En ambos grupos, aunque principalmente entre las/os trabajadores de casas particulares, hay una muy elevada proporción de mujeres (ANSES, 2020).

Otro dato interesante que surge de la inscripción al programa es la gran cantidad de solicitantes no bancarizados, incluso entre aquellos que estuvieron o están en relación de dependencia. En este punto es importante recordar que la obligación de los empleadores de bancarizar a los trabajadores rige en Argentina desde hace décadas.

Una vez realizada la inscripción de los solicitantes el Estado nacional realizó una evaluación patrimonial y socioeconómica de las personas inscriptas a fin de corroborar la situación de real necesidad del individuo y de su grupo familiar. Tal como señalan Arcidiácono y Gamallo (2020) se estableció un esquema probatorio frecuente en las políticas de transferencia de ingresos y estructuradas sobre criterios focalizadores con el objetivo de detectar incompatibilidades mediante dispositivos burocráticos orientados a distinguir a los beneficiarios legítimos e ilegítimos y desalentando el comportamiento de los "pícaros" con el recurso de los cruces de bases de información ${ }^{13}$.

Así, 4,6 millones de personas solicitantes del IFE no fueron seleccionadas a partir de esta evaluación realizada. Entre las razones del rechazo del beneficio es posible mencionar: a) por tener él, ella o alguna persona de su grupo familiar un empleo en relación de dependencia (2 millones de personas, 43,6\%); b) los/as solicitantes -o algún integrante de su grupo familiar- posee ingresos por jubilaciones o pensiones $(0,7$ millones, $15,6 \%)$; c) eran titulares -o alguien de su grupo familiar- de algún plan social incompatible $(0,3$ millones, $6,4 \%)$; d) no superaron el control socioeconómico o de gastos (0,3 millones, $6,0 \%)$; e) eran monotributistas categoría $\mathrm{C}$ o superior $(0,2$ millones, $5,0 \%$; f) ya había otro/a beneficiario/a en su grupo familiar que percibía la AUH y se le asignó el IFE a esa persona ( 0,2 millones, $4,5 \%)$ y g) eran extranjeros/ as con datos insuficientes (0,2 millones, 4,4\%) (ANSES, 2020).

Así, y para Arcidiácono y Gamallo (2020), la principal razón de denegación se vinculó con el criterio combinado entre atributos individuales y de los miembros

12 El programa PROGRESAR es una beca que se otorga a jóvenes de 18 a 24 años que no tienen empleo, trabajan informalmente o perciben un salario menor al mínimo vital y móvil y su grupo familiar se encuentre en iguales condiciones, con el objetivo que puedan iniciar o concluir sus estudios en cualquier nivel.

13 Los criterios focalizadores y los procedimientos burocráticos de selección se afinaron poco tiempo después, estableciéndose nuevos criterios que regirían para el segundo pago del programa. Un ejemplo de eso es la exclusión del pago a las personas privadas de la libertad y el cambio de criterio para los menores de 25 años que sólo podrían acceder al beneficio si el domicilio de residencia registrado en la Administración Nacional de Seguridad Social fuera diferente al de sus padres (durante la inscripción al programa bastaba que la persona solicitante menor a 25 años acreditara un domicilio diferente a sus padres para que se lo considerara grupo familiar unipersonal). 
de la unidad convivencial, en especial la situación laboral y los ingresos, de acuerdo con el registro que ANSES tenía de dicha composición. Esa condición es un aspecto frecuente de inclusión y exclusión de las prestaciones sociales en las transferencias no contributivas.

Finalmente, es importante señalar que el programa se implementó con celeridad y articulando distintos organismos del Estado como el Ministerio de Economía, el Ministerio de Trabajo, Empleo y Seguridad Social, el Ministerio de Desarrollo Social, el Banco Central de la República Argentina, la Administración Federal de Ingresos Públicos, la Jefatura de Gabinete de Ministros y la Administración Nacional de Seguridad Social, organismo que administra, otorga, paga, controla y supervisa la correcta liquidación del beneficio.

Se realizaron tres pagos del IFE (originariamente estaba pautado un único pago): el primero entre abril y mayo de 2020, el segundo entre junio y julio y el último en agosto y septiembre. El primer pago supuso transferencias por 0,3\% del PBI y el costo fiscal total al terminar el tercer pago llegó a ARS 270 millones de pesos lo que representa el 0,9\% del PBI.

\section{Las características de los y las beneficiarias del IFE y sus impactos}

Según el informe elaborado por ASES al finalizar el primer pago, la población beneficiaria fue de casi 8,9 millones de personas, las cuales pueden dividirse en dos grandes grupos:

1. IFE 1: corresponde a la población beneficiaria de la AUH o AE. Se trata de una población de 2.389.764 personas, de las cuales casi el 95\% son mujeres. A este grupo las transferencias llegaron de manera automática e inmediata ya que se trata de una población sobre la cual el Estado cuenta con información y está bancarizada. Ese conjunto concentró ayudas extraordinarias (bonos y la Tarjeta Alimentar), en especial aquellas familias con menores de seis años: una familia con dos hijos de tal edad percibió en abril \$22.000 (adición de montos correspondientes al IFE, al bono AUH y a la Tarjeta Alimentar), alcanzando $\$ 28.200$ al agregarse $\$ 6.200$ correspondientes al pago regular de la AUH (Arcidiácono y Gamallo, 2020).

2. IFE 2: incorporó a 6,5 millones de personas, distribuidos entre unos 700 mil monotributistas, 180 mil trabajadoras de casas particulares, 128 mil receptores del plan PROGRESAR y 5,5 millones de personas trabajadoras informales o desocupadas (de los cuales 3,3 millones son varones).

De esta información de los perceptores del IFE se desprenden dos consideraciones centrales, una en términos de género y la segunda en torno del sector informal en el mercado de trabajo argentino. 


\section{Tabla N 1}

Composición de la población receptora del IFE

\begin{tabular}{|l|c|c|c|}
\hline Composición & Total IFE & Titulares AUH & No titulares AUH \\
\hline Varones & $44,30 \%$ & $5,30 \%$ & $59 \%$ \\
\hline Mujeres & $55,70 \%$ & $94 \%$ & $41 \%$ \\
\hline Total & $\mathbf{1 0 0} \%$ & $\mathbf{1 0 0} \%$ & $\mathbf{1 0 0} \%$ \\
\hline
\end{tabular}

Fuente: Oficina Nacional de Presupuesto, Ministerio de Economía y Dirección Nacional de Coordinación del Presupuesto Nacional, Jefatura de Gabinete (2020)

La mayor presencia femenina entre los perceptores del IFE puede explicarse por una conjunción de varios factores. El primero está dado por el sesgo administrativo - establecido en el diseño de la política- de privilegiar esa titularidad en caso de más de un solicitante por hogar. El segundo factor refiere a la inclusión automática de perceptores de AUH o AE entre los beneficiarios del IFE. En este punto es fundamental señalar que por las características propias de la AUH más del 90\% de los titulares son mujeres. Algo similar acontece con los y las jóvenes perceptores de la beca PROGRESAR, donde la presencia femenina sobrepasa el 67\%.

Vinculado a este punto -y en relación a la situación laboral- la inclusión de las y los trabajadores de casas particulares constituye un tercer factor explicativo, ya que se corresponde con el sector más feminizado de la economía. La participación femenina en este sector supera el 93\%, presentando además altísimos niveles de no registro (78\% de los y las trabajadores del sector se encuentran en esta situación según datos del INDEC) y perciben el salario promedio más bajo en el mercado de trabajo.

Si bien estos tres elementos aparecen como centrales para explicar la mayor presencia femenina entre los beneficiarios del IFE, otros componentes colaboran en esa explicación porque de hecho solo en una de las modalidades o situaciones laborales recabadas al momento de la inscripción al programa éste presenta mayor presencia de varones. 
Tabla N 2

Perceptores IFE, según modalidad/situación laboral 2020

\begin{tabular}{|l|c|c|}
\hline Modalidad/Situación Laboral & Perceptores & \% de mujeres \\
\hline AUH (IFE 1) & 2.389 .764 & $94,90 \%$ \\
\hline Monotributistas & 688.556 & $45,10 \%$ \\
\hline PROGRESAR & 128.201 & $67,4 \%$ \\
\hline Trabajadores de Casas Particulares & 188.923 & $97 \%$ \\
\hline Informales/Desocupados & 5.461 .617 & $61,80 \%$ \\
\hline Otras & 2 & $\mathrm{n} / \mathrm{a}$ \\
\hline Total & $\mathbf{8 . 8 5 7 . 0 6 3}$ & $\mathbf{5 5 , 7 0 \%}$ \\
\hline
\end{tabular}

Fuente: ANSES, 2020

El dato más destacado cuando se observa la discriminación de perceptores por modalidad/situación laboral es el de la alta presencia de trabajadores y trabajadoras informales y desocupados/as, que representan el 61,7\% de los perceptores. Sin lugar a dudas en este punto se anudan las dos cuestiones centrales de análisis: la problemática de género y la del trabajo informal.

La focalización en los trabajadores informales era un objetivo central de la política ya que el establecimiento de medidas generalizadas de aislamiento social constituía un duro golpe para este grupo de trabajadores y trabajadoras que no disponen de un salario fijo ni de ahorros y para los cuales buena parte de su reproducción cotidiana depende de las vicisitudes de una actividad económica fundada básicamente en una perpetua y constante adaptación a la demanda. Para esos sectores la irregularidad de sus trabajos se corresponde con la irregularidad de sus ingresos, los cuales -sumados a la ausencia de seguridades y protecciones- construyen un marco cotidiano caracterizado por la precariedad. Así, las condiciones de trabajo se desarrollan en un contexto de ausencia de certezas respecto de la subsistencia (Delfino, 2015).

En este grupo la proporción de mujeres que recibió las transferencias también fue mayor que la proporción de varones. Históricamente, tanto en Argentina como a nivel global, las mujeres se insertan en el mercado de trabajo de forma diferente y desigual en relación a los varones, lo que se manifiesta en tasas de desempleo, de subempleo y de precariedad más elevadas y en sectores de la economía con menores salarios. Estas tendencias además tienden a agudizarse en períodos como los descriptos al inicio de estas páginas, donde es posible observar cómo el mercado de trabajo argentino se encontraba en una situación de acumulación de tensiones de largo plazo incluso antes del inicio de la pandemia. 
En esta dirección y para el caso argentino es posible señalar que el mercado de trabajo femenino confirma las pautas históricas de comportamiento en el marco de una crisis. La primera es que de las crisis se sale con una intensificación del trabajo de las mujeres, incluyendo el trabajo remunerado y, sobre todo, el no remunerado. La segunda pauta supone que tras la crisis, el empleo masculino se recupera siempre antes que el femenino y este último acaba siempre aún más precarizado que cuando se inicia la crisis; y la tercera que de las crisis se sale con retrocesos en los avances en igualdad conseguidos en épocas de bonanza en lo relativo a la regulación, las políticas de igualdad y las reglas de juego en general (Gálvez Muñoz y Rodríguez Modroño, 2012).

Respecto a los grupos de edad, la mayor incidencia del desempleo y de la informalidad entre los jóvenes dio como resultado que el 33,3\% de las transferencias se concentraran en el grupo entre los 25 y 34 años, seguidas por el $28,2 \%$ de las transferencias direccionadas a jóvenes entre 18 y 24 años. Así, el 52\% de los jóvenes de entre 18 y 24 años y el 44\% de los de 25 a 34 fueron alcanzados por la prestación (ANSES, 2020).

Vinculado a los jóvenes surge otro dato interesante a ser señalado. Se estimó que el $22 \%$ de los hogares recibió el IFE. Excluyendo a los hogares beneficiarios de AUH, el 63\% (4,1 millones) se declararon unipersonales, entre los cuales 1,7 millones están compuestos por menores de 24 años (ANSES, 2020). Tal como señalan Arcidiácono y Gamallo (2020) la magnitud de los hogares unipersonales beneficiarios es llamativamente inconsistente con la información censal, ya que según el Censo Nacional de Población, Hogares y Viviendas 2010, el país contaba con 2,1 millones de hogares de ese tipo (incluyendo a aquellos con mayores de 65 años). Para los autores estos datos generan dudas sobre el criterio de categorización utilizado.

Para finalizar podemos señalar que el programa analizado surgió por la necesidad de brindar apoyo económico a los sectores más vulnerables buscando asegurar el confinamiento en los barrios populares. De los datos de su implementación es posible desprender tres consideraciones. La primera refiere a que el universo de sus beneficiarios y beneficiarias permite observar de forma clara la extensión de la informalidad y la precariedad del mercado de trabajo en Argentina. Apenas un diez por ciento había gozado de un empleo formal en relación de dependencia durante el año previo. Este dato es central, ni siquiera el Estado nacional había podido estimar el tamaño de la población objeto de la política por desconocimiento; lo que es lo mismo que decir que el Estado desconoce quiénes son, cuántos son y cómo sobreviven las poblaciones más vulnerables que habitan su territorio. También equivale a decir, como ya lo señalaron Arcidiácono y Gamallo (2020), que entre los beneficiarios existían un buen número de personas que reclamaron a la ventanilla de asistencia estatal por primera vez en su trayectoria personal. 
Una segunda consideración está vinculada con la noción que la asimetría y desigualdad de las relaciones que presiden una sociedad de clases son también constitutivas de las relaciones de género y en esta dirección el género guarda una relación integral con el sistema de estratificación por clase.Así, la división social del trabajo se asienta sobre las líneas de género demarcadas por la división sexual (Ariza y de Oliveira, 1999).

Un tercer elemento de análisis da cuenta que aun con la magnitud que tuvieron las transferencias, las mismas fueron insuficientes para absorber la totalidad del impacto del aislamiento social en el mercado de trabajo. La caída del empleo fue un factor excluyente del incremento de la población bajo la línea de pobreza que pasó de 35 a 40\% en el segundo semestre de 2019 y el primero de 2020 (Kennedy, 2020). Esas cifras continuaron aumentando a lo largo de 2020, alcanzando la pobreza el 42\%, mientras que la indigencia trepó al 10,5\% según datos del INDEC.

\section{A manera de cierre}

A partir de la Modernidad, el trabajo se constituye tanto en la base principal de la cual se derivan las condiciones materiales de vida de la población como en el sustento al que se ligan las protecciones contra la inseguridad. De forma conjunta, el trabajo supuso la sujeción de los individuos al orden social a través de la interiorización de dispositivos disciplinares. Es en torno a esta idea moderna que se articuló lo central del sistema de seguridad en Argentina desde mediados del siglo XX.

Tal como señala Repetto $(2001,2007)$, serían tres las áreas sustantivas de la política social como función del Estado. El primer conjunto de intervenciones contiene aquellas definidas como políticas sociales universales. Refiere a aquellos servicios que el Estado decide que debe prestar por razones históricas y/o por su importancia estratégica en diferentes sectores. La educación básica y la atención primaria de salud constituyen los exponentes centrales de este tipo de intervención.

El segundo grupo estaría constituido por las políticas de seguridad social; es decir, aquellas protecciones cuya cobertura se estructura en función del empleo formal. Su financiamiento es asegurado por el aporte de los trabajadores, los empleadores y el Estado y los beneficios que otorgan están parcialmente vinculados a esos aportes.

La última región o área sustantiva de la política social son las políticas asistenciales o políticas frente a la pobreza. La lógica funcional de ésta se pone de manifiesto cuando el Estado presta atención a categorías de riesgo que no entran en las "regiones" anteriores: los sectores sociales que no pueden proveerse ingresos por el mercado de trabajo y que, por diferentes razones, a pesar de existir provisión pública de servicios no pueden acceder a ella. La política asistencial sería de todas las ramas de la política social, aquella identificada por la intervención sobre grupos de mayor riesgo relativo.

En los momentos en los cuales el sistema de seguridad social se consolida, el crecimiento económico permitía pensar a ese sistema como casi universal, en tanto 
el mercado de trabajo mostraba indicadores cercanos al pleno empleo. La asistencia social aparecía como una intervención social residual en ese marco. De forma clara, las construcciones modernas en torno al trabajo y los trabajadores estaban en el fundamento de la estructura que tomaban las intervenciones del Estado en estas áreas.

Pero esa situación comenzó a cambiar paulatinamente a partir de la última dictadura militar. La estructura del mercado de trabajo se transformó y el patrón de distribución del ingreso se tornó crecientemente regresivo. La crisis socio-económica-política de 2001 fue un verdadero parte aguas. A partir de allí comenzaron a diseñarse una serie de programas de empleo transitorio y de políticas de transferencias condicionadas como forma de atención a la población que se encontraba en los márgenes del mercado de trabajo.

Sin embargo, los años de crecimiento económico no lograron revertir la situación: permaneciendo una tasa de informalidad próxima al 30\% y un núcleo duro de pobreza y marginalidad que las políticas desplegadas no lograron penetrar. En ese marco se crea la AUH destinada a los hijos de las familias excluidas del empleo formal y por primera vez su gestión se inscribió en el sistema de seguridad social.

Los datos del Ingreso Familiar de Emergencia diseñado y ejecutado durante la pandemia están mostrando la necesidad y la urgencia de repensar los esquemas de protecciones de forma tal que permitan otorgar certidumbres inter-temporales a los grupos sociales vulnerables. Lo Vuolo (2020) señala que lo que parece como un problema de coyuntura no debe observarse como un elemento aislado sino como uno que profundiza problemas estructurales de larga data y expone la asimétrica vulnerabilidad de los distintos grupos sociales de una sociedad cada vez más desigual.

Le queda a la sociedad y al Estado en la etapa de la pospandemia una tarea de gran magnitud: pensar y estructurar un nuevo sistema de protección social que cumpla un rol integrador ya perdido. 


\section{Referencias}

ANSES (2020). Boletín IFE I - 2020: Caracterización de la población beneficiaria. Julio.

Arcidiácono, P. y Gamallo, G. (2020). El Ingreso Familiar de Emergencia. Respuesta inmediata y debates futuros. Serie de Debates $N^{\circ} 2$. Grupo de Trabajo Interdisciplinario. Recuperado el 15 de marzo de 2021 de: https://www.dspp.com.ar/wp-content/ uploads/DSP-2007010-Debates-02-IFE-Arcidiacono-y-Gamallo.pdf

Ariza, M. y de Oliveira, O. (1999). Inequidades de género y clase: algunas consideraciones analíticas. Nueva Sociedad, 164, noviembre-diciembre, 70-81.

Astarita, M. y De Piero, S. (2017). "Cambiemos y una nueva forma de elitismo: el políticoempresarial”, en Daniel García Delgado y Agustina Gradin (coordinadores). Documento de trabajo $N^{\circ}$ 5, El Neoliberalismo tardío:Teoría y Praxis. Buenos Aires: FLACSO

Barrera Insua, F. y Pérez, P. (2019). “Como comer y descomer: Flexibilización laboral y baja salarial durante el gobierno de Cambiemos". En Pablo Belloni y Francisco Cantamutto (Coords.).La economía política de Cambiemos: Ensayos sobre un nuevo ciclo neoliberal en la Argentina. Ciudad Autónoma de Buenos Aires: Batalla de Ideas.

Benza, G. y Kessler, G. (2021). La ¿Nueva? estructura social de América Latina: cambios y persistencias después de la ola de gobiernos progresistas. $1^{\text {a }}$ ed. Buenos Aires: Siglo Veintiuno Editores.

Campana, J. y. Ferrari Mango, C. (2021). "El Estado entre desigualdades y políticas públicas redistributivas. A un año de la crisis sanitaria". Informe N 19 del Observatorio sobre Políticas Públicas y Reforma Estructural, FLACSO: Buenos Aires.

CEPAL (2020). Dimensionar los efectos del COVID-19 para pensar en la reactivación. Informe Especial COVID-19, $N^{\circ} 2,21$ de abril.

CETyD (2020a). Políticas sociolaborales en tiempos del COVID-19. Cobertura y desafios futuros. Buenos Aires: Instituto de Altos Estudios Sociales, Universidad Nacional de San Martín.

CETyD (2020b). El Impacto de la pandemia sobre un mercado laboral vulnerable. Buenos Aires: Instituto de Altos Estudios Sociales, Universidad Nacional de San Martín.

CIFRA (2017). El balance laboral de los dos primeros años del gobierno de Cambiemos: menos empleo, menor registración, menores salarios. Buenos Aires: CIFRA.

CIFRA (2020). Informe sobre empleo y salarios registrados. Diciembre 2020. Recuperado el 2 de marzo de 2021 en: http://www.centrocifra.org.ar/publicacion.php?pid=160. 
CIFRA-FLACSO (2016) “La naturaleza política y económica de la alianza Cambiemos”, en Documento de trabajo, Buenos Aires, No 15. Recuperado el 21 de marzo de 2021 en:: http://www.centrocifra.org.ar/docs/PL.pdf

Delfino, A. (2015). Programas de transferencias monetarias condicionadas y temporalidad social. Un análisis del Programa Jefes y Jefas de Hogar Desocupados y su incidencia en el uso del tiempo de la población beneficiaria. Tesis de Doctorado. Doctorado en Ciencia Política. Universidad Nacional de Rosario. Directora: Marta Panaia, Codirectora: Adriana Chiroleu.

Delfino, A. y Kaplan, L. (2019). Reforma integral vs. reforma "por partes": todos los caminos conducen a la flexibilización. Un análisis de las transformaciones en la institucionalidad laboral durante la gestión de Cambiemos (2015-2018), en Esteban Iglesias y Juan Bautista Lucca (comp), La Argentina de Cambiemos. Rosario: Editorial Universidad Nacional de Rosario.

Díaz Langou, G.; De León, G.; Florito, J.; Caro Sachetti, F.; Biondi Rodríguez, A. y Karczmarczyk, M. (2019). El género del trabajo: Entre la casa, el sueldo y los derechos. Buenos Aires: CIPPEC, OIT, ONU Mujeres, PNUD.

Dirección Nacional de Economía, Igualdad y Género (2020). Ingreso Familiar de Emergencia. Análisis y desafios para la transferencia de ingresos a trabajadores/as precarios/as. Ministerio de Economía. Secretaría de Política Económica. Dirección Nacional de Economía, Igualdad y Género. 27/04/2020.

Ernst, C. y López Mourelo, E. (2020). El COVID-19 y el mundo del trabajo en Argentina: impacto y respuestas de política. Informe Técnico. Buenos Aires: Oficina de País de la OIT para la Argentina

Gálvez Muñoz, L. y Rodríguez Modroño, P. (2012) ."La desigualdad de género en las crisis económicas”. Investigaciones Feministas,Vol. 2, pp. 113-132. Disponible en: https://doi. org/10.5209/rev_INFE.2011.v2.38607

INDEC (2021), Mercado de trabajo. Tasas e indicadores socioeconómicos (EPH). Cuarto trimestre de 2020. Informes técnicos Vol. 5, n52. Recuperado el 1 de abril de 2021 en: https://www.indec.gob.ar/uploads/informesdeprensa/mercado_trabajo_ eph_4trim20126C4AD8D8.pdf

Kennedy, D. (2020) "Algunas reflexiones en torno a las perspectivas generales del mercada de trabajo argentino pospandemia”.Voces del Fénix, año 10, Nro. 82, diciembre.

Lo Vuolo, R. (2020) “La Argentina frente al shock asimétrico", Voces del Fénix, año 10, Nº 82 , diciembre.

Manzanelli, P.; Calvo, D. y Basualdo, E. (2020). Un balance preliminar de la crisis económica en Argentina. Documento de Trabajo N17. CIFRA-FLACSO 
Minujín, Alberto y Cosentino, Estela (1993), "Crisis y futuro del estado de bienestar. Aportes a un debate”, en Minujín, Alberto (Ed.) Desigualdad y Exclusión. UNICEF/Losada, Buenos Aires.

MTEySS (abril de 2021). Situación y evolución del mercado de trabajo. Datos a marzo de2021.http://www.trabajo.gob.ar/downloads/estadisticas/trabajoregistrado/ trabajoregistrado_2003_informe.pdf.

Oficina Nacional de Presupuesto, Ministerio de Economía y Dirección Nacional de Coordinación del Presupuesto Nacional, Jefatura de Gabinete (2020), Seguimiento del gasto vinculado con políticas de género en el presupuesto nacional. Primer trimestre 2020. Disponible en: https://www.presupuestoabierto.gob.ar/sici/listado-informesgenero

Pastrana, F. y Trajtemberg, D. (2020). Salarios y empleos en el laberinto de la pandemia. Revista La Nación Trabajadora. Recuperado el 22 de abril de 2021 en: https:// lanaciontrabajadora.com/ensayo/laberinto-salarial/.

Repetto, F. (2001), Gestión pública y desarrollo social en los noventa: Las trayectorias de Argentina y Chile. Buenos Aires: Prometeo.

Repetto, F. (2007), "Nueva matriz socio-política, problemas sociales y políticas públicas. América Latina a inicios del Siglo XXI" en Gonzalo Saraví (Ed.). De la pobreza a la exclusión: continuidades y rupturas de la cuestión social en América Latina. Buenos Aires: Prometeo.

Salvia, A. y Vera, J. (2015). Las desigualdades estructurales y el efecto de la educación sobre las oportunidades de empleo pleno. En Hora de Balance: Proceso de acumulación, mercado de trabajo y bienestar. Argentina, 2002-2014. Buenos Aires: EUDEBA.

Tokman, V. (1997). De la informalidad a la modernidad. Santiago de Chile: Organización Internacional del Trabajo (OIT).

UMET-CITRA (2019). El rol del monotributo en la precarización laboral. Informe $\mathrm{N}^{\circ}$ 15. Recuperado el 5 de abril de 2021 en: https://pulsocitra.org/wp-content/ uploads/2019/01/CEPE-15.pdf

Wainfeld, M. (2020). “El desconocido mundo de los más humildes” Página 12, 20 de abril.

Enviado: 04 de mayo de 2021

Aceptado: 02 de julio de 2021 